\title{
Intrinsic intensity fluctuations in random lasers
}

\author{
Karen L. van der Molen, ${ }^{1, *}$ Allard P. Mosk, ${ }^{1}$ and Ad Lagendijk ${ }^{2}$ \\ ${ }^{1}$ Complex Photonic Systems, MESA ${ }^{+}$Research, Institute and Department of Science and Technology, \\ University of Twente, P.O. Box 217, 7500 AE Enschede, The Netherlands \\ ${ }^{2}$ FOM Institute for Atomic and Molecular Physics (AMOLF), Kruislaan 407, 1098 SJ Amsterdam, The Netherlands
}

(Received 16 May 2006; published 15 November 2006)

\begin{abstract}
We present a quantitative experimental and theoretical study of intensity fluctuations in the emitted light of a random laser that has different realizations of disorder for every pump pulse. A model that clarifies these intrinsic fluctuations is developed. We describe the output versus input power graphs of the random laser with an effective spontaneous emission factor ( $\beta$ factor).
\end{abstract}

DOI: 10.1103/PhysRevA.74.053808

PACS number(s): 42.55.Zz, 42.25.Dd

\section{INTRODUCTION}

In 1968 Letokhov wrote his pioneering paper [1] in which he predicted that light amplification through stimulated emission is possible in a random medium with gain. A preeminent experimental demonstration of such a random laser was published by Lawandy et al. in 1994 [2]. Typical phenomena of the random laser are a (low) threshold in the power conversion, spectral narrowing, and sharp features ("spikes") in the emitted spectrum for both picosecond [3-6] and nanosecond [7] pump pulses. Due to their low threshold and their ease of production, random lasers are expected to be used in many applications, such as coding of clothing [8] and detection of dangerous materials [9]. The theoretical search for the underlying principles of random lasers intensifies, focusing on their statistical properties and fluctuations [10-13].

Recently, a new random-laser phenomenon was described by Anglos et al. [14]. They observed shot-to-shot intensity fluctuations in the emitted light, which were not caused by fluctuations in the pump source. In their experiments, the intensity fluctuations occur for nanosecond pump pulses, but not for picosecond pump pulses. The physical understanding of the underlying principle of these fluctuations is relevant for applications and our perception of random lasers.

In this paper we present a quantitative experimental and theoretical study of the statistics of these shot-to-shot intensity fluctuations of random laser systems that have different realizations of disorder for every pump pulse, and develop a model that clarifies the existence of these fluctuations. Our aim is to provide physical understanding of the fluctuations. With such a model, experimental conditions can be tailored to control the fluctuations.

This paper is organized as follows. In Sec. II the experimental setup is described, followed in Sec. III by the experimental observations. A model based on the number of laser modes in the random laser is presented in Sec. IV. We compare our model with experimental observations in Sec. V.

\section{APPARATUS AND SAMPLES}

We start by describing the experimental details of our samples and the setup. The random laser consists of a sus-

\footnotetext{
*Electronic address: k.l.vandermolen@utwente.nl
}

pension of $\mathrm{TiO}_{2}$ particles (mean diameter of $180 \mathrm{~nm}$ ) in a solution of methanol and Sulforhodamine B $(1 \mathrm{mmol} / \mathrm{liter})$. The suspension is contained in a fused silica capillary tube, with internal dimensions $100 \times 2 \times 0.2 \mathrm{~mm}^{3}$. To characterize the mean free path of this sample, we performed an enhanced-backscatter cone experiment [15] and an escape function experiment [16]. We found a transport mean free path of $0.46 \pm 0.1 \mu \mathrm{m}$ at $\lambda=633 \mathrm{~nm}$.

The samples are excited by a pump pulse at $532 \mathrm{~nm}$, provided by an optical parametric oscillator (OPO) pumped by a $Q$-switched Nd:YAG laser (Coherent Infinity 40-100/XPO). The pump pulse has a duration of $3 \mathrm{~ns}$ and a repetition rate of $50 \mathrm{~Hz}$. The pump light is focused with a microscope objective (water-immersed, numerical aperture $\mathrm{NA}=1.2$ ) onto the sample (focus area $=12 \pm 6 \mu \mathrm{m}^{2}$ ), reaching an intensity in the order of $1 \mathrm{~mJ} / \mathrm{mm}^{2}$. The light emitted by the random laser is collected by the same objective. A small part of the pump light is split off from the input beam before the objective. This light is used to excite a dye solution (Cresyl Violet) which we use as a marker for the pump fluence. This dye solution works as a wavelength converter for the pump light. The light emitted by Cresyl Violet and by the random laser are recorded at the same time with a spectrometer and an intensified charged coupled device (resolution $\sim 0.3 \mathrm{~nm}$ spectral width): in one frame both the emission of the random laser and the pump marker is recorded. The pump light is filtered out of the detection path by use of a colored glass filter with a transmission of less than $1 \%$ at the wavelength of the pump laser.

As a picosecond source we use a stretched pulse at $532 \mathrm{~nm}$ of a femtosecond optical parametric amplifier (OPA), pumped by a femtosecond Ti:sapphire laser. We could not measure the pump pulse duration directly, but calculated a duration of $15 \pm 0.5$ ps from the measured bandwidth of the OPA and the configuration of the pulse stretcher. Measurements of the random laser and Cresyl Violet simultaneously were not performed with the picosecond pump pulse.

\section{MEASURED FLUCTUATIONS}

We are interested in the intrinsic fluctuations, i.e., the fluctuations of the random laser itself which are not the result of fluctuations of the pump laser. A typical single shot emission spectrum shows two peaks, see the inset of Fig. 1: the 


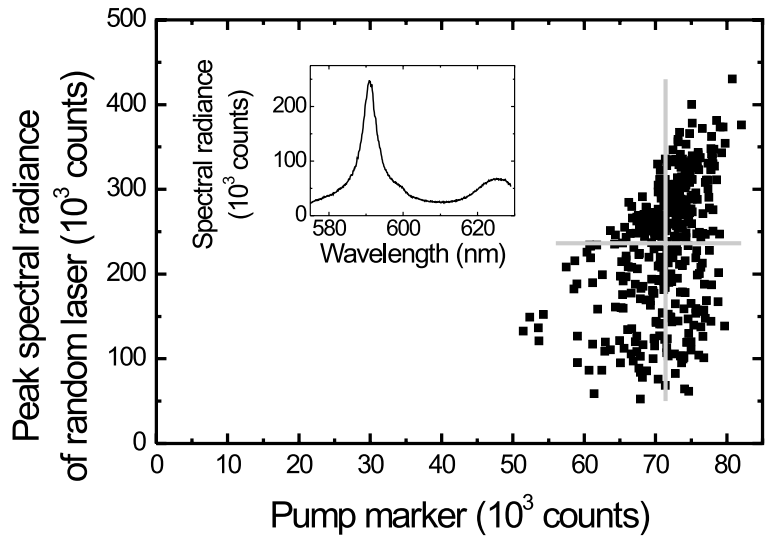

FIG. 1. The peak of the spectral radiance of the random laser is plotted vs the pump marker. The two gray lines indicate the mean values of the two peak heights and are used to visualize the correlation. The inset shows a typical single shot spectrum, from which the counts of the peak heights are determined.

peak of the random laser output is around $594 \mathrm{~nm}$, and the peak of the marker Cresyl Violet is around $625 \mathrm{~nm}$. The intrinsic fluctuations of the random laser are investigated by comparing the peak height of the light emitted by the random laser with that of the marker. We have taken many single shot measurements $(>400)$, and from each of these spectra we determine the two peak heights. The data are shown in Fig. 1. The corresponding correlation coefficient is 0.4 , indicating that a large part of the fluctuations is intrinsic. In contrast, we expect stronger correlation between the spontaneous emission regime of the random laser and the pump fluence. To check this expectation we compute the correlation coefficient for the spectral radiance at $573 \mathrm{~nm}$, relatively far away from the random laser emission peak, and the pump marker. For this situation we find a stronger correlation coefficient of 0.62 , indicating that these fluctuations are mainly due to the pump fluctuations. Although the spectral radiance at $573 \mathrm{~nm}$ is still partially influenced by stimulated emission, the effect of the spontaneous emission can already be seen from the increase of the correlation coefficient.

The intrinsic fluctuation coefficient $f$ is defined as the ratio between the standard deviation of the shot-to-shot intensity of the light emitted by the random laser, $\Delta I$, and the mean value of the intensity of the emitted light $\bar{I}$,

$$
f \equiv \frac{\Delta I}{\bar{I}} .
$$

To determine the intrinsic fluctuations from the data points in Fig. 1 we take a small band of pump fluences so that the fluctuations of the pump laser do not influence the outcome. We make a histogram of the accompanying peak heights of the spectral radiance of the random laser and fit this histogram with a Gaussian. From this fit we obtain the standard deviation and the mean value of the peak heights, leading us to the experimental fluctuations

$$
f_{\text {ns,exp }}=(18 \pm 3) \%,
$$

where the error margin corresponds to one standard deviation.
Due to large technical fluctuations of the pump laser, no reliable estimate of the intrinsic fluctuations follows from the picosecond pumped random laser experiments.

\section{MODEL}

In this section we present a model for the origin of the intrinsic fluctuations of random lasers. The approach we take here is based on the concept of pseudomodes [17]. Pseudomodes are single frequency eigenmodes, solutions of Maxwell equations. These modes have an eigenfrequency and decay by leaking to the outside world. This leakage is characterized by a decay time $\tau_{c}$. Each mode can be a laser mode, depending on the decay time (also referred to as dwell time) of that mode and the gain time of the sample. The decay time is the time that light is inside the sample due to diffusion, and the amplitude gain time is defined as the time after which the amplitude is increased with a factor $e$. If the decay time of a certain pseudomode is longer than the gain time of the system, that pseudomode is a laser mode. We assume the mode volume is equal for each (random) mode, and that the gain in the sample is homogeneous. The number of lasing modes, $N_{l}$, is given by

$$
N_{l}=\pi_{l} N,
$$

with $N$ the number of pseudomodes and $\pi_{l}$ is a random variable ranging between 0 and 1 . We define

$$
p_{l} \equiv \bar{\pi}_{l}
$$

with $p_{l}$ the probability for lasing in a pseudomode, and $\bar{\pi}_{l}$ is $\pi_{l}$ averaged over realizations of the disorder. The emission power of the different lasing modes is equal. The detected emission intensity of the random laser is almost entirely due to the lasing modes in the system and is assumed to be proportional to the number of lasing modes. The intrinsic fluctuations $f$ can be determined

$$
f=\frac{\Delta I}{\bar{I}}=\frac{\sqrt{\bar{N}_{l}}}{\overline{N_{l}}}
$$

In the last step of Eq. (5), we have assumed a binomial distribution of $N_{l}$, which results for the limit of $N$ to infinity to a Gaussian or normal distribution. The standard deviation, $\sigma$, is given by $\sqrt{\bar{N}_{l}}$. We combine Eqs. (3) and (4), and insert the result in Eq. (5) to obtain

$$
f=\frac{1}{\sqrt{p_{l} N}} .
$$

In Sec. IV A we will show how to calculate $p_{l}$ from a fit to experimental data. An elaboration on the calculation of $N$ is presented in Sec. IV B.

\section{A. Determination of the probability of lasing}

When we combine Eqs. (3) and (4) the probability of lasing is given by 


$$
p_{l}=\frac{\overline{N_{l}}}{N} \text {. }
$$

The probability of lasing can be calculated via the distribution of the decay times. The integral of the distribution of the decay times $P$ from the gain time to infinity will give the probability $p_{l}$ for a certain mode to be a laser mode,

$$
p_{l}=\int_{\tau_{g}}^{\infty} P\left(\tau_{c}\right) d \tau_{c} .
$$

The distribution of the decay times for a three dimensional (3D) diffusive medium is not known. We therefore use the distribution of the phase delay times [18], which is expected to be close to the distribution of the decay times.

The gain time is given by

$$
\tau_{g} \equiv \frac{\ell_{g} n^{\prime}}{c_{0}},
$$

where $n^{\prime}$ is the real part of the refractive index. The amplitude gain length $\ell_{g}$ is given by

$$
\ell_{g}=\frac{2}{\sigma_{e} \rho_{\mathrm{exc}}},
$$

where $\sigma_{e}$ is the stimulated emission cross section of a molecule, and $\rho_{\text {exc }}$ is the density of molecules in the excited state in the sample.

If the pump power is large enough, the gain in the system can be saturated. In the case of saturation the gain length will not be decreased any more when the pump power is increased. From Eq. (10) we can find a lower bound for the gain length $\ell_{g, b}$, and thus an indication of gain saturation, when one assumes that all the dye molecules in the medium are in the excited state

$$
\ell_{g, b} \geq \frac{2}{\sigma_{e} \rho}
$$

where $\rho$ is the density of dye molecules in the sample.

We want to determine the probability of lasing directly from our experiments. When we examine Eq. (7), we see a similarity between the definition of $p_{l}$ and the spontaneous emission factor of a laser, the $\beta$ factor [19]. The single-mode $\beta$ factor, defined as the fraction of spontaneous emission that contributes to lasing, is given by [20]

$$
\beta_{\mathrm{sm}}=\frac{1}{N} \text {. }
$$

This $\beta_{\mathrm{sm}}$ factor appears in the four-level rate equations for a single-mode laser [20]

$$
\begin{gathered}
\frac{d N_{1}(t)}{d t}=P_{L}(t)-\frac{\beta_{\mathrm{sm}} q(t) N_{1}(t)}{\tau}-\frac{N_{1}(t)}{\tau}, \\
\frac{d q(t)}{d t}=-\frac{q(t)}{\tau_{c}}+\frac{\beta_{\mathrm{sm}} N_{1}(t)}{\tau}[q(t)+1],
\end{gathered}
$$

with $N_{1}$ the number of excited molecules in the medium, $q$ the number of photons in the lasing mode, $P_{L}$ the pump rate (in photons per second), $\tau$ the spontaneous emission lifetime of the dye, and $\tau_{c}$ the cavity decay time.

In a random laser many random modes contribute to the laser oscillation. However, for our consideration only the average behavior is relevant. In general, to describe a multimode laser one has to write an equation for every mode and couple the different mode equations to the equation for the population. Only two small changes to the single-mode rate equations are necessary, if the behavior of the multimode laser can be simulated by the behavior of a single-mode laser. We simply replace $\beta_{\mathrm{sm}}$ and $\tau_{c}$ in the rate equations (13) by the effective parameters $\beta_{\mathrm{mm}}$ and $\tau_{c, \mathrm{~mm}}$. Since we are interested in the average behavior, we will use this simplified approach. We will prove that this approach is valid in many situations.

For the mean value of the cavity decay time, given by $\tau_{\mathrm{c}, \mathrm{mm}}$, we use the mean value of the distribution of the decay times. To determine this distribution we calculate the solution of the diffusion equation for a slab with thickness $L$, with a source positioned in the middle of the slab. From this solution of the diffusion equation the electric field correlation is derived and the mean value of the phase delay times follows from the Taylor expansion of this correlation [21]. The mean value of the phase delay time, and thus the mean value of the cavity decay time, is given by

$$
\tau_{c, \mathrm{~mm}}=\frac{1}{8} \frac{L^{2}}{D} .
$$

The diffusion constant $D$ is given by $c_{0} \ell /\left(3 n^{\prime}\right)$, with $\ell$ the transport mean free path [22]. For the effective parameter $\beta_{\mathrm{mm}}$ we take

$$
\beta_{\mathrm{mm}}=\frac{\overline{N_{l}}}{N}
$$

where we assumed that all modes contribute equally. Comparing Eqs. (7) and (15) we see that $\beta_{\mathrm{mm}}$ is equal to $p_{l}$.

In the continuous wave limit for a single-mode laser in steady state a formula can be analytically derived from the rate equations (13) that describes the relation between the output and the input power of a laser,

$$
q=\frac{1}{2} \sqrt{\left(\frac{1-P_{L} \tau_{c} \beta_{\mathrm{sm}}}{\beta_{\mathrm{sm}}}\right)^{2}+4 P_{L} \tau_{c}}-\frac{1-P_{L} \beta_{\mathrm{sm}} \tau_{c}}{2 \beta_{\mathrm{sm}}} .
$$

If one uses a pulsed pump this should be generalized to include time dependence, and an analytic solution is no longer available. However, as we will show, for a wide range of parameters Eq. (16) still describes the threshold behavior very well, even for a detector that integrates the output power. We will use Eq. (16) for the integrated power of a multimode laser, with the replacements of the parameters as described above. For the use of Eq. (16) in pulsed experiments we replace the parameter $\beta_{\mathrm{mm}}$ by an effective parameter $\beta_{\text {eff. }}$.

To examine the applicability of Eq. (16) to experiments with a pulsed pump, we calculate with the rate equations (13) several output versus input power graphs. We use input parameters relevant to our experiment and vary the pump pulse duration and $\beta_{\mathrm{sm}}$. The pump pulse is modeled by a Gaussian. 


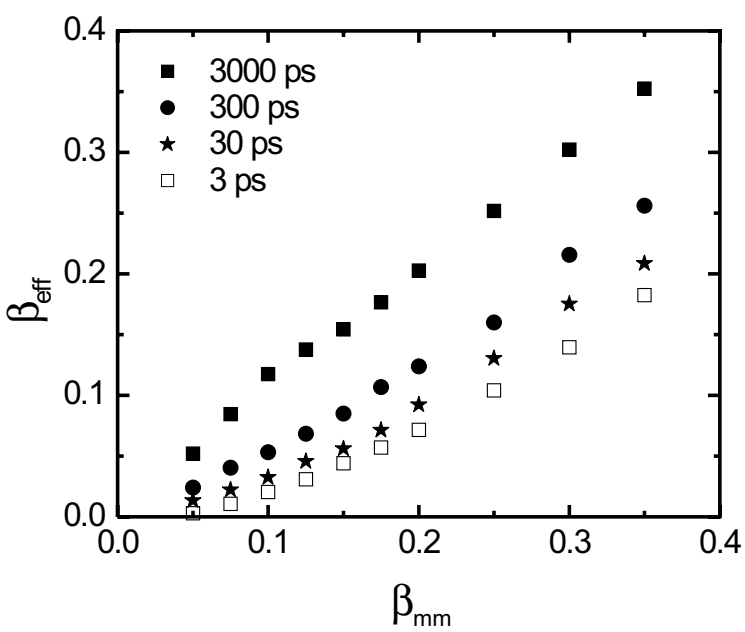

FIG. 2. The theoretical values of the effective $\beta$ factor, $\beta_{\text {eff }}$, numerically calculated from the rate equations, vs the input parameter $\beta_{\mathrm{mm}}$ for different pump pulse durations. We choose the input parameters relevant for our experiments: $\tau=3200 \mathrm{ps}$ and $\tau_{c}$ $=0.1 \mathrm{ps}$. In the legend the pump pulse durations are listed.

To the output versus input power graphs we fit Eq. (16) and use as fit parameter $\beta_{\text {eff. }}$. In Fig. 2 we present the calculated $\beta_{\text {eff }}$ as a function of $\beta_{\mathrm{mm}}$. For a pulse duration $t_{p}$ of $3000 \mathrm{ps,}$ the calculated values of $\beta_{\text {eff }}$ are identical to the input value of $\beta_{\mathrm{mm}}$. This correspondence implies that for our system nanosecond pump pulses can be treated as a continuous wave pump. When the pulse duration is shorter, while keeping the other parameters constant, we notice a deviation from this straight line to lower values of $\beta_{\text {eff }}$ for the same values of $\beta_{\mathrm{mm}}$. This difference between $\beta_{\text {eff }}$ and $\beta_{\mathrm{mm}}$ increases for shorter pulses. The origin of the dissimilarity between $\beta_{\mathrm{mm}}$ and $\beta_{\text {eff }}$ is due to the fact that the pump is not a continuous wave, but a pulse with a finite duration.

Besides the pump pulse duration and the spontaneous lifetime, there is a third time scale in the rate equations: the mean cavity decay time. If the pump pulse duration approaches the value of the cavity decay time $\tau_{c, \mathrm{~mm}}$, Eq. (16) is no longer a good fit to the output versus input power graph. This failure of the fit means that $\beta_{\text {eff }}$ is no longer a parameter that can be used to describe the experiment and thus that for that experiment there is no $\beta$ factor. In short:

$$
\begin{gathered}
t_{p} \geq \tau \gg \tau_{c}, \quad \text { CW limit: } \beta_{\mathrm{eff}}=\beta_{\mathrm{mm}}, \\
\tau>t_{p}>\tau_{c}, \quad \text { conversion needed: } \beta_{\mathrm{eff}}<\beta_{\mathrm{mm}}, \\
\tau>\tau_{c}>t_{p}, \quad \text { simplified model fails. }
\end{gathered}
$$

We conclude that the rate equations and the threshold curve (16) can be used for a multimode laser, and that these formulas can be used under certain conditions for a pulsed laser. This allows us to extract $p_{l}$ from the threshold curve of a random laser.

\section{B. Calculation of the number of modes}

The second important parameter to calculate the intrinsic fluctuations is $N$. The total number of modes in the system

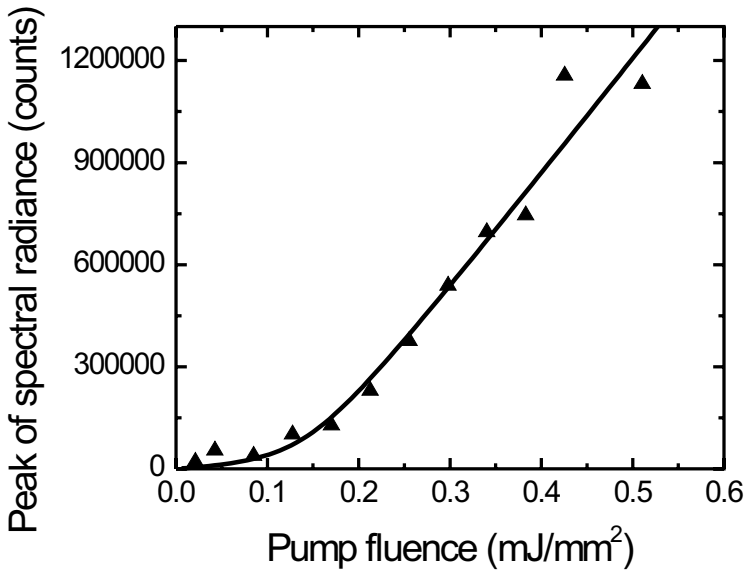

FIG. 3. Peak of the measured spectral radiance of the random laser vs the pump fluence for a random laser pumped with nanosecond pulses. A normal threshold behavior is observed. The solid line is a fit to our data, with $\tau_{c \text {,mm }}=0.1 \mathrm{ps}$, and the spontaneous emission lifetime is 3200 ps. From the fit we obtain $\beta_{\text {eff }}=0.07$, which in our model equals the probability of lasing $p_{l}$ [Eq. (16)].

within the relevant frequency bandwidth $\Delta \omega$ can be calculated using the formula [20]

$$
\begin{aligned}
N & =\rho(\omega, V) \frac{\Delta \omega}{\omega} \\
& =\frac{8 \pi n^{3} V}{\lambda_{c}^{3}} \frac{\Delta \lambda}{\lambda_{c}},
\end{aligned}
$$

with $\rho(\omega, V)$ the density of field modes in the cavity volume $V, \Delta \lambda$ the full width at half maximum of the emission spectrum, and $\lambda_{c}$ the central wavelength of the emission spectrum. All parameters can be deduced from experiments, except the volume of the cavity $V$. In our case, the absorption length is much larger than the transport mean free path, and we can assume a gain volume in the form of a hemisphere

$$
V=\frac{2}{3} \pi r^{3}
$$

with $r$ the radius of the gain volume.

The intrinsic fluctuations depend on the number of modes, and the probability of lasing. By experimentally varying the quantities of the parameters, the intrinsic fluctuations of the random laser can be controlled.

\section{RESULTS}

We will now calculate with our model the intrinsic intensity fluctuations of a random laser system pumped with nanosecond or picosecond pulses, and compare these fluctuations with experimental observations.

\section{A. Probability of lasing}

We have measured the peak of the spectral radiance of the random laser as a function of the pump fluence, as shown in Fig. 3. In case of nanosecond pump pulses we have seen that 


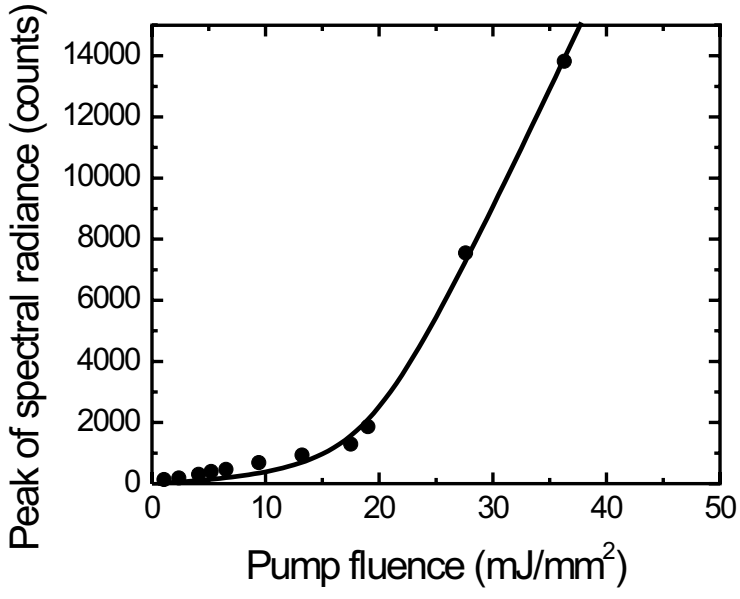

FIG. 4. Peak of the measured spectral radiance vs pump fluence for a random laser pumped with picosecond pulses. The solid line is a fit to our data, with the parameters $\tau=3200 \mathrm{ps}$ and the mean cavity decay time $0.1 \mathrm{ps}$. From the fit we obtain $\beta_{\mathrm{eff}}=0.03$ [Eq. (16)].

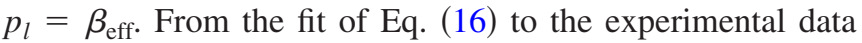
we find directly the probability of lasing

$$
p_{l, \mathrm{~ns}}=0.07 \pm 0.03 \text {, }
$$

where the error margin corresponds to one standard deviation.

For the picosecond pumped random laser we have also measured the peak of the spectral radiance of the random laser as a function of the pump fluence, see Fig. 4. The parameter $\beta_{\text {eff }}$ has to be converted to $\beta_{\mathrm{mm}}\left(p_{l}\right)$. In our picosecond experiment the pulse duration is $15 \pm 0.5 \mathrm{ps}$ and a relevant conversion graph for $\beta_{\mathrm{mm}}$ and $\beta_{\mathrm{eff}}$ is presented in Fig. 5. With this graph we convert our values of $\beta_{\text {eff }}$ of 0.03 \pm 0.006 to

$$
p_{l, \mathrm{ps}}=0.09 \pm 0.015 \text {, }
$$

where the error margin corresponds to one standard deviation.

\section{B. Number of modes}

The calculation of the number of modes for our random laser regime is given by Eq. (18). In our case we have $n$ $=1.4837, \lambda_{c}=595 \mathrm{~nm}$. The width of the emission spectrum above threshold is $4.3 \mathrm{~nm}$. The volume of the gain medium is given by the volume of a hemisphere, see Eq. (19). The spatial form of the luminescence coming from the surface of the random laser sample pumped with nanosecond pump pulses was recorded with a charge coupled device, while we filter the pump light. We measure a circular spot with a mean radius of $5 \pm 0.5 \mu \mathrm{m}$. The number of modes in the nanosecond pumped situation is

$$
N_{n s}=746 \pm 256 .
$$

We did not record the spatial form of the luminescence in case of picosecond pump pulses. However we can speculate about the gain volume in the picosecond pumped system if we have gain saturation. For our random laser, the probabil-

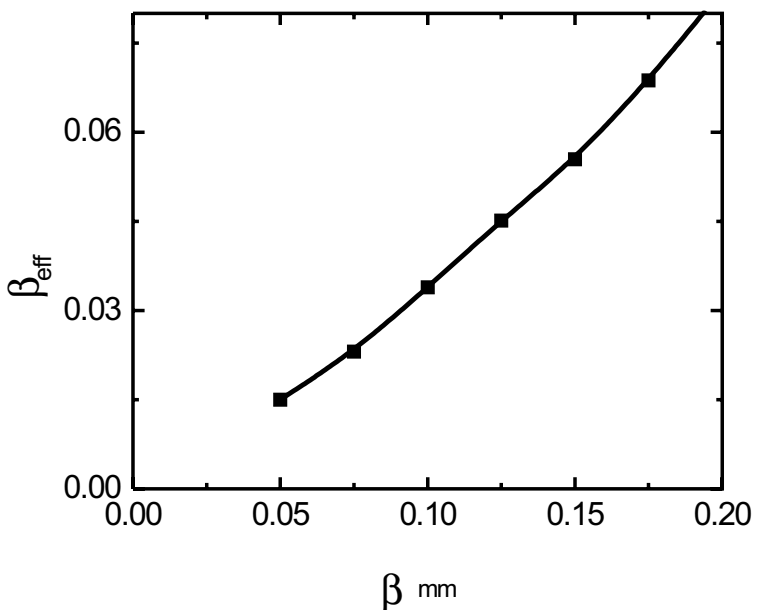

FIG. 5. The calculated $\beta_{\text {eff }}$ vs the input parameter of the rate equations $\beta_{\mathrm{mm}}$ is shown. The graph is produced for a pump pulse duration of $15 \mathrm{ps}, \tau_{c, \mathrm{~mm}}=0.1 \mathrm{ps}$, and $\tau=3200 \mathrm{ps}$. The solid lines connect the data points. We convert our $\beta_{\text {eff }}$ of 0.03 to a $p_{l}$ of 0.09 .

ity of lasing for the picosecond and for the nanosecond pumped case are within each other's error margins, an indication of gain saturation which we shall prove now.

We calculate the lower bound of the gain length with Eq. (11). The density of molecules in the random laser is 5.4 $\times 10^{23}$ molecules $\mathrm{m}^{-3}$, and the stimulated emission cross section is $4 \times 10^{-20} \mathrm{~m}^{2}$, both with a $10 \%$ error, leading to a lower bound limit of

$$
\ell_{g, b} \geq 75 \mu \mathrm{m}
$$

For the nanosecond pumped random laser we found a $p_{l}$ of $0.07 \pm 0.03$, see Fig. 3. The gain length at which $p_{l}$ equals 0.04 is

$$
\ell_{g, b, c}=60 \mu \mathrm{m}
$$

The value of $\ell_{g, b, c}$ is lower than the bound limit we calculated. Although this does not indicate a serious discrepancy as the error margins on $p_{l}$ are only one standard deviation, the assumption that the phase delay time distribution equals the cavity decay time distribution should be investigated. Both gain lengths above are almost equal, proving that we are near the saturation regime. The threshold pump fluence is a factor 100 higher in the case of the picosecond pump pulse, and the pulse duration of the picosecond pump pulse is a factor 100 shorter. Since both pump lasers pump the sample to gain saturation, the gain volume of the picosecond pump pulse is larger than the gain volume of the nanosecond pump pulse. The advantage of using two different pump lasers on one sample is the change in the number of laser modes.

The gain volume for the picosecond case in saturation is at minimum a cylinder with a radius equal to the luminescence spot of the nanosecond pumped random laser and a length equal to $L_{d}$. The length $L_{d}$ is the length that light travels from a point source inside a diffusive medium 


$$
L_{d}=\sqrt{D t},
$$

with $t$ the pulse duration of the point source. In our system $L_{d}$ is $22 \pm 3 \mu \mathrm{m}$. This leads to a total number of modes in the picosecond pumped random laser of

$$
N_{p s} \geq 2407 \pm 885 \text {. }
$$

\section{Intrinsic intensity fluctuations of a random laser}

The intensity fluctuations derived from our model are given by Eq. (5)

$$
f_{n s}=(14 \pm 5) \%,
$$

where the error margin corresponds to one standard deviation. This number for the fluctuations is in good agreement with our experimental observations of $(18 \pm 3) \%$.

The intrinsic fluctuations calculated for a picosecond pump pulse for our own system is

$$
f_{p s} \leq(6.8 \pm 1.2) \%,
$$

where the error margin corresponds to one standard deviation. We could not verify this result experimentally. Anglos et al. have performed measurements on a random laser pumped with picosecond pulses [14]. From their paper we can calculate the fluctuations of their system with our model. From the fit to the published output versus input power graph, and with a conversion from $\beta_{\text {eff }}$ to $p_{l}$, we find for their system $p_{l}=0.05$. For the gain volume we assumed a cylindrical form, as their excitation spot is much larger than their mean free path. We obtain $f_{p s}=0.01 \%$. From their published measured spectra we can find an estimation for the measured fluctuations. The measured fluctuations are $(3.7 \pm 1.7) \%$, where the error margin corresponds to one standard deviation. These fluctuations include the pump fluctuations. These pump fluctuations can be in the order of $3 \%$ for a typical picosecond laser source. Since the expected intrinsic fluctuations are much smaller than the pump fluctuations, the intrinsic fluctuations cannot be measured and our model is not inconsistent with their data.

\section{CONCLUSIONS}

We have developed a model based on quasimodes that predicts the fluctuations of the output power of a random laser pumped with either nanosecond or picosecond pulses. For the system pumped with nanosecond pulses we computed fluctuations of $(14 \pm 5) \%$. This is in good agreement with our experimental fluctuations of $(18 \pm 3) \%$. For a system pumped with picosecond pulses we calculated $f_{p s}=0.01 \%$, too small to be observed, for the system measured by Anglos et al. [14].

The difference in intrinsic fluctuations between picosecond and nanosecond pumped random lasers is well described by our model and the predictions are identical to our observations for a nanosecond pumped random laser and published observations for a picosecond pumped random laser. Our model can be used to tailor experimental conditions in such a way as to control the intrinsic fluctuations of a random laser system.

\section{ACKNOWLEDGMENTS}

We thank Boris Bret for the discussion that led to the estimation of the transport mean free path, Tom Savels for fruitful discussions considering the model, and Tijmen Euser and Willem Vos for use of the femtosecond OPA system. This work is part of the research program of the Stichting voor Fundamenteel Onderzoek der Materie (FOM), which is financially supported by the Nederlandse Organisatie voor Wetenschappelijk Onderzoek (NWO).
[1] V. S. Letokhov, Sov. Phys. JETP 26, 835 (1968).

[2] N. M. Lawandy, R. M. Balachandran, A. S. L. Gomes, and E. Sauvain, Nature (London) 368, 436 (1994).

[3] H. Cao, Y. G. Zhao, H. C. Ong, S. T. Ho, J. Y. Dai, J. Y. Wu, and R. P. H. Chang, Appl. Phys. Lett. 73, 3656 (1998).

[4] S. Mujumdar, M. Ricci, R. Torre, and D. S. Wiersma, Phys. Rev. Lett. 93, 053903 (2004).

[5] R. C. Polson, M. E. Raikh, and Z. V. Vardeny, IEEE J. Sel. Top. Quantum Electron. 9, 120 (2003).

[6] V. Milner and A. Z. Genack, Phys. Rev. Lett. 94, 073901 (2005).

[7] S. V. Frolov, Z. V. Vardeny, A. A. Zakhidov, and R. H. Baughmann, Opt. Commun. 162, 241 (1999).

[8] H. Ramachandran, Pramana, J. Phys. 58, 313 (2002).

[9] A. Rose, Z. Zhu, C. F. Madigan, T. M. Swager, and V. Bulovic, Nature (London) 434, 876 (2005).

[10] L. I. Deych, Phys. Rev. Lett. 95, 043902 (2005).

[11] L. Angelani, C. Conti, G. Ruocco, and F. Zamponi, Phys. Rev. Lett. 96, 065702 (2006).

[12] A. Yamilov, X. Wu, H. Cao, and A. Burin, Opt. Lett. 30, 2430 (2005).

[13] D. Sharma, H. Ramachandran, and N. Kumar, Opt. Lett. 31,
1806 (2006).

[14] D. Anglos, A. Stassinopoulos, R. N. Das, G. Zacharakis, M. Psyllaki, R. Jakubiak, R. A. Vaia, E. P. Giannelis, and S. H. Anastasiadis, J. Opt. Soc. Am. B 21, 208 (2004).

[15] M. P. Van Albada and A. Lagendijk, Phys. Rev. Lett. 55, 2692 (1985).

[16] J. Gomez Rivas, D. H. Dau, A. Imhof, R. Sprik, B. P. J. Bret, P. M. Johnson, T. W. Hijmans, and A. Lagendijk, Opt. Commun. 220, 17 (2003).

[17] Y. Ling, H. Cao, A. L. Burin, M. A. Ratner, X. Liu, and R. P. H. Chang, Phys. Rev. A 64, 063808 (2001).

[18] B. A. van Tiggelen, P. Sebbah, M. Stoytchev, and A. Z. Genack, Phys. Rev. E 59, 7166 (1999).

[19] J. P. Woerdman, M. P. van Exter, and N. J. van Druten, Adv. At., Mol., Opt. Phys. 47, 205 (2001).

[20] A. E. Siegman, Lasers (Standford University, University Science Books, Oxford, 1986).

[21] A. Z. Genack, P. Sebbah, M. Stoytchev, and B. A. van Tiggelen, Phys. Rev. Lett. 82, 715 (1999).

[22] P. Sheng, Introduction to Wave Scattering, Localization, and Mesoscopic Phenomena (Academic Press, San Diego, 1995). 